\title{
Asymptotics of Orthogonal Polynomials via Recurrence Relations
}

\author{
X.-S. Wang* and R. Wong ${ }^{\dagger}$
}

\begin{abstract}
We use the Legendre polynomials and the Hermite polynomials as two examples to illustrate a simple and systematic technique on deriving asymptotic formulas for orthogonal polynomials via recurrence relations. Another application of this technique is to provide a solution to a problem recently raised by M. E. H. Ismail.
\end{abstract}

Keywords Asymptotics; orthogonal polynomials; recurrence relation; Legendre Polynomials; Hermite Polynomials.

AMS Subject Classification Primary 41A60 · Secondary 33C45

\footnotetext{
${ }^{*}$ Corresponding author. Department of Mathematics and Statistics, York University, Toronto, Ontario, Canada

${ }^{\dagger}$ Department of Mathematics, City University of Hong Kong, Tat Chee Avenue, Kowloon, Hong Kong
} 


\section{Introduction}

There are many powerful and systematically developed techniques in asymptotic theory for orthogonal polynomials. For instance, the steepest-descent method for integrals [9], the WKB (Liouville-Green) approximation for differential equations [7], the Deift-Zhou's nonlinear steepest-descent method for RiemannHilbert problems [2, 3], and etc. Here, we intend to develop a simple, and yet systematic approach to derive asymptotic formulas for orthogonal polynomials by using their recurrence relations. Let $\left\{\pi_{n}(x)\right\}_{n=0}^{\infty}$ be a system of monic polynomials satisfying the recurrence relation

$$
\pi_{n+1}(x)=\left(x-a_{n}\right) \pi_{n}(n)-b_{n} \pi_{n-1}(x), \quad n \geq 1,
$$

and the initial conditions $\pi_{0}(x)=1$ and $\pi_{1}(x)=x-a_{0}$. Note that for the sake of convenience, we have normalized the polynomials to be monic. To construct the asymptotic formulas of $\pi_{n}(x)$, we first set

$$
\pi_{n}(x)=\prod_{k=1}^{n} w_{k}(x) .
$$

It is readily seen from (1.1) that $w_{1}(x)=x-a_{0}$ and

$$
w_{k+1}(x)=x-a_{k}-\frac{b_{k}}{w_{k}}, \quad k \geq 1 .
$$

When $x$ is away from the oscillatory region of the orthogonal polynomials, it is easy to find an asymptotic formula for $w_{k}(x)$ from (1.3) . Then, as we shall see, the asymptotic behavior of $\pi_{n}(x)$ for $x$ away from the oscillatory region can be obtained readily. When $x$ is near the oscillatory region, we use a method similar to that given in [8, 10, to derive asymptotic formulas for general solutions of (1.1). The asymptotic formula of $\pi_{n}(x)$ for $x$ near the oscillatory region is then obtained by doing a matching. In the subsequent three sections, we will consider the following three cases:

Case 1: $a_{n}=0$ and $b_{n}=n^{2} /\left(4 n^{2}-1\right)$. This case is related to the Legendre polynomials.

Case 2: $a_{n}=0$ and $b_{n}=n / 2$. This case is related to the Hermite polynomials.

Case 3: $a_{n}=n^{2}$ and $b_{n}=1 / 4$. This case was recently brought to our attention by M. E. H. Ismail.

For simplicity, we use the same notations in the following three sections. Because each section is independent and self-contained, this will not lead to any confusion.

\section{Case 1: the Legendre polynomials}

The Legendre polynomials can be defined as [6, (1.8.57)]

$$
P_{n}(x)={ }_{2} F_{1}\left(\begin{array}{c|c}
-n, n+1 & \frac{1-x}{2}
\end{array}\right) .
$$

They satisfy the recurrence relation [6, (1.8.59)]

$$
(2 n+1) x P_{n}(x)=(n+1) P_{n+1}(x)+n P_{n-1}(x) .
$$

For convenience, we normalize the Legendre polynomials to be monic. Put

$$
\pi_{n}(x):=\frac{2^{n} n !}{(n+1)_{n}} P_{n}(x) .
$$

The monic Legendre polynomials $\left\{\pi_{n}(x)\right\}_{n=0}^{\infty}$ satisfy [6, (1.8.60)]

$$
\begin{aligned}
& \pi_{n+1}(x)=x \pi_{n}(x)-\frac{n^{2}}{4 n^{2}-1} \pi_{n-1}(x), \quad n \geq 1, \\
& \pi_{0}(x)=1, \quad \pi_{1}(x)=x .
\end{aligned}
$$


Theorem 2.1. As $n \rightarrow \infty$, we have

$$
\pi_{n}(x) \sim\left(\frac{x+\sqrt{x^{2}-1}}{2}\right)^{n}\left(\frac{x+\sqrt{x^{2}-1}}{2 \sqrt{x^{2}-1}}\right)^{1 / 2}
$$

for $x$ in the complex plane bounded away from $[-1,1]$.

Proof. Set

$$
\pi_{n}(x)=\prod_{k=1}^{n} w_{k}(x)
$$

From (2.1), (2.2) and (2.4), it follows that

$$
\begin{aligned}
& w_{k+1}(x)=x-\frac{k^{2}}{4 k^{2}-1} \frac{1}{w_{k}(x)}, \quad k \geq 1, \\
& w_{1}(x)=x .
\end{aligned}
$$

As $k \rightarrow \infty$, we have

$$
w_{k}(x) \sim \frac{x+\sqrt{x^{2}-1}}{2}
$$

for $x \in \mathbb{C} \backslash[-1,1]$. Here the square root takes its principle value so that $\sqrt{x^{2}-1} \sim x$ as $x \rightarrow \infty$. Define

$$
w(x):=\frac{x+\sqrt{x^{2}-1}}{2}
$$

and

$$
u_{k}(x):=\frac{w_{k}(x)}{w(x)} .
$$

It is easily seen from (2.5), (2.6) and (2.8) that

$$
\begin{aligned}
& u_{k+1}(x)=\frac{x}{w(x)}-\frac{k^{2}}{4 k^{2}-1} \frac{1}{w(x)^{2} u_{k}(x)}, \quad k \geq 1, \\
& u_{1}(x)=\frac{x}{w(x)} .
\end{aligned}
$$

We make a change of variable

$$
t=t(x):=\left(x-\sqrt{x^{2}-1}\right)^{2} .
$$

It follows from (2.7) and (2.11) that

$$
w(x)^{2}=\frac{1}{4 t}, \quad \frac{x}{w(x)}=1+t .
$$

Hence, the equations (2.9) and (2.10) can be written as

$$
\begin{aligned}
& u_{k+1}(x)=1+t-\frac{4 k^{2} t}{4 k^{2}-1} \frac{1}{u_{k}(x)}, \quad k \geq 1, \\
& u_{1}(x)=1+t
\end{aligned}
$$


Define $Q_{0}(t):=1$ and

$$
Q_{n}(t):=\prod_{k=1}^{n} u_{k}(x), \quad n \geq 1 .
$$

From (2.13), (2.14) and (2.15), we obtain $Q_{1}(t)=1+t$ and

$$
Q_{n+1}(t)=(1+t) Q_{n}(t)-\frac{4 n^{2} t}{4 n^{2}-1} Q_{n-1}(t) .
$$

From this recurrence relation, one can construct a generating function from which it is easily deducible that $Q_{n}(t)$ has the explicit expression

$$
Q_{n}(t)=\sum_{j=0}^{n} \frac{(1 / 2)_{j}(n-j+1)_{j}}{j !(n-j+1 / 2)_{j}} t^{j}
$$

A simpler verification of this identity is by induction. Using the Lebesgue dominated convergence theorem, it can be readily shown that

$$
Q_{n}(t) \rightarrow(1-t)^{-1 / 2}
$$

as $n \rightarrow \infty$. Note that by (2.4), (2.8) and (2.15), $\pi_{n}(x)=w(x)^{n} Q_{n}(t)$. Thus, it follows that

$$
\pi_{n}(x) \sim w(x)^{n}(1-t)^{-1 / 2}
$$

as $n \rightarrow \infty$. This together with (2.7) and (2.11) yields (2.3).

Theorem 2.2. Let $\delta>0$ be any fixed small number. For $x$ in a small complex neighborhood of the interval $[-1+\delta, 1-\delta]$, we have

$$
\pi_{n}(x) \sim \frac{1}{2^{n}}\left[\cos n \theta\left(\frac{1+\sin \theta}{\sin \theta}\right)^{1 / 2}+\sin n \theta\left(\frac{1-\sin \theta}{\sin \theta}\right)^{1 / 2}\right]
$$

as $n \rightarrow \infty$, where $\theta=\theta(x):=\arccos x$ with $0<\operatorname{Re} \theta<\pi$.

Proof. To put the difference equation (2.1) in the form suggested by Wang and Wong [8, (2.1)], we let

$$
p_{n}(x):=\frac{2^{n} \Gamma(n / 2+1 / 4) \Gamma(n / 2+3 / 4)}{[\Gamma(n / 2+1 / 2)]^{2}} \pi_{n}(x) .
$$

From (2.17), it is easily seen that

$$
\frac{[\Gamma(n / 2+1 / 2)]^{2}(n / 2+1 / 4)}{[\Gamma(n / 2+1)]^{2}} \cdot 2 x p_{n}(x)=p_{n+1}(x)+p_{n-1}(x) .
$$

Motivated by the form of the normal (series) solutions to second-order difference equations (see [10, (1.5)]), we assume

$$
p_{n}(x) \sim n^{\alpha}[r(x)]^{n}\{f(x) \cos [n \varphi(x)]+g(x) \sin [n \varphi(x)]
$$

as $n \rightarrow \infty$, where $r(x)$ and $\varphi(x)$ are real-valued functions, whereas $f(x)$ and $g(x)$ can be complex-valued. We now proceed to determine the constant $\alpha$ and the functions $r(x), \varphi(x), f(x)$ and $g(x)$ in (2.19). It can be easily shown from (2.19) that

$$
p_{n \pm 1}(x) \sim n^{\alpha} r^{n \pm 1}[(f \cos \varphi \pm g \sin \varphi) \cos (n \varphi)+(g \cos \varphi \mp f \sin \varphi) \sin (n \varphi)] .
$$


Furthermore, by the asymptotic formula for the ratio of Gamma functions [1, (6.1.47)] we have

$$
\frac{[\Gamma(n / 2+1 / 2)]^{2}(n / 2+1 / 4)}{[\Gamma(n / 2+1)]^{2}}=1+O\left(n^{-2}\right)
$$

as $n \rightarrow \infty$. Applying (2.19), (2.20) and (2.21) to (2.18) gives

$$
\begin{aligned}
2 x[f \cos (n \varphi)+g \sin (n \varphi)] \sim & r[(f \cos \varphi+g \sin \varphi) \cos (n \varphi)+(g \cos \varphi-f \sin \varphi) \sin (n \varphi)] \\
& +r^{-1}[(f \cos \varphi-g \sin \varphi) \cos (n \varphi)+(g \cos \varphi+f \sin \varphi) \sin (n \varphi)] .
\end{aligned}
$$

Comparing the coefficients of $\cos (n \varphi)$ and $\sin (n \varphi)$ on both sides of the last formula yields

$$
\begin{gathered}
2 x f=\left(r+r^{-1}\right) f \cos \varphi+\left(r-r^{-1}\right) g \sin \varphi ; \\
2 x g=-\left(r-r^{-1}\right) f \sin \varphi+\left(r+r^{-1}\right) g \cos \varphi .
\end{gathered}
$$

Thus, we obtain from the above two equations

$$
x=\cosh (\log r) \cos \varphi, \quad 0=\sinh (\log r) \sin \varphi .
$$

It can be easily seen that the only solution to these equations is $\log r=0$ and $\varphi=\arccos x$. Recall that

$$
\theta=\theta(x):=\arccos x, \quad 0<\operatorname{Re} \theta<\pi .
$$

Hence, we conclude that

$$
r=1 \quad \text { and } \quad \varphi=\theta .
$$

Next we are going to determine the constant $\alpha$ in (2.19). Applying (2.23) to (2.19) gives

$$
p_{n}(x) \sim n^{\alpha}[f \cos (n \theta)+g \sin (n \theta)],
$$

and

$$
p_{n \pm 1}(x) \sim n^{\alpha}\left(1 \pm \frac{\alpha}{n}\right)[(f \cos \theta \pm g \sin \theta) \cos (n \theta)+(g \cos \theta \mp f \sin \theta) \sin (n \theta)]
$$

A combination of (2.18), (2.21), (2.24) and (2.25) yields

$$
2 x[f \cos (n \theta)+g \sin (n \theta)] \sim 2 f \cos \theta \cos (n \theta)+2 g \cos \theta \sin (n \theta)+\frac{\alpha}{n}[2 g \sin \theta \cos (n \theta)-2 f \sin \theta \sin (n \theta)] .
$$

In view of (2.22), we obtain by matching the coefficients in the last formula

$$
\alpha g \sin \theta=0, \quad \alpha f \sin \theta=0 .
$$

These equations hold for all $x$ in a small complex neighborhood of $[-1+\delta, 1-\delta]$. Since $f$ and $g$ can not be identically zero, it follows that

$$
\alpha=0 .
$$

Thus, we have from (2.19), (2.23) and (2.26)

$$
p_{n}(x) \sim f \cos n \theta+g \sin n \theta
$$

as $n \rightarrow \infty$. This formula holds uniformly for $x$ in a small complex neighborhood of $[-1+\delta, 1-\delta]$. Moreover, it follows from (2.3) and (2.17) that

$$
p_{n}(x) \sim\left(x+\sqrt{x^{2}-1}\right)^{n}\left(\frac{x+\sqrt{x^{2}-1}}{2 \sqrt{x^{2}-1}}\right)^{1 / 2}
$$


for complex $x$ bounded away from $[-1,1]$. Our last step is to determine the coefficients $f$ and $g$ in (2.27) by matching the above two formulas in an overlapping region. With $\theta$ and $x$ given by (2.22), it can be shown that for $\operatorname{Im} x>0$, we have $\operatorname{Im} \theta<0$. Thus, (2.27) implies

$$
p_{n}(x) \sim\left(\frac{f}{2}+\frac{g}{2 i}\right) e^{i n \theta} .
$$

Meanwhile, in view of $x=\cos \theta$ and $\sqrt{x^{2}-1}=i \sin \theta$ by (2.22), we obtain from (2.28) that

$$
p_{n}(x) \sim e^{i n \theta}\left[\frac{e^{i(\theta-\pi / 2)}}{2 \sin \theta}\right]^{1 / 2}
$$

Coupling the last two formulas gives

$$
\frac{f}{2}+\frac{g}{2 i}=\frac{e^{i(\theta / 2-\pi / 4)}}{(2 \sin \theta)^{1 / 2}}
$$

Similarly, matching (2.27) with (2.28) in the region $\operatorname{Im} x<0$ yields

$$
\frac{f}{2}-\frac{g}{2 i}=\frac{e^{-i(\theta / 2-\pi / 4)}}{(2 \sin \theta)^{1 / 2}}
$$

From the last two equations of $f$ and $g$ we have

$$
f=\left(\frac{1+\sin \theta}{\sin \theta}\right)^{1 / 2}, \quad g=\left(\frac{1-\sin \theta}{\sin \theta}\right)^{1 / 2} .
$$

This together with (2.17) and (2.27) implies (2.16).

\section{Case 2: the Hermite polynomials}

The Hermite polynomials can be defined as [․ (1.13.1)]

$$
H_{n}(x)=(2 x)_{2}^{n} F_{0}\left(\begin{array}{c|c}
-n / 2,-(n-1) / 2 & -\frac{1}{x^{2}}
\end{array}\right) .
$$

They satisfy the recurrence relation [ $\underline{6},(1.13 .3)]$

$$
2 x H_{n}(x)=H_{n+1}(x)+2 n H_{n-1}(x) .
$$

For convenience, we normalize the Hermite polynomials to be monic, and put

$$
\pi_{n}(x):=2^{-n} H_{n}(x) .
$$

The monic Hermite polynomials $\left\{\pi_{n}(x)\right\}_{n=0}^{\infty}$ satisfy [6, (1.13.4)]

$$
\begin{aligned}
& \pi_{n+1}(x)=x \pi_{n}(x)-\frac{n}{2} \pi_{n-1}(x), \quad n \geq 1, \\
& \pi_{0}(x)=1, \quad \pi_{1}(x)=x .
\end{aligned}
$$

Theorem 3.1. As $n \rightarrow \infty$, we have

$$
\pi_{n}(\sqrt{2 n} y) \sim\left(\frac{n}{2 e}\right)^{n / 2} \exp \left\{n\left[y^{2}-y \sqrt{y^{2}-1}+\log \left(y+\sqrt{y^{2}-1}\right)\right]\right\}\left(\frac{y+\sqrt{y^{2}-1}}{2 \sqrt{y^{2}-1}}\right)^{1 / 2}
$$

for complex y bounded away from the interval $[-1,1]$. 
Proof. Set

$$
\pi_{n}(x)=\prod_{k=1}^{n} w_{k}(x)
$$

It follows from (3.1) and (3.2) that $w_{1}(x)=x$ and

$$
w_{k+1}(x)=x-\frac{k}{2 w_{k}(x)} .
$$

Let $x=x_{n}:=\sqrt{2 n} y$ with $y \in \mathbb{C} \backslash[-1,1]$. It can be proved by induction that for real $y$ and $y \notin[-1,1]$, we have

$$
\frac{x_{n}+\sqrt{x_{n}^{2}-2 k}}{2}\left[1+\frac{1}{2\left(x_{n}^{2}-2 k\right)}-\frac{5 x_{n}-\sqrt{x_{n}^{2}-2 k}}{8\left(x_{n}^{2}-2 k\right)^{5 / 2}}\right]<w_{k}\left(x_{n}\right)<\frac{x_{n}+\sqrt{x_{n}^{2}-2 k}}{2}\left[1+\frac{1}{2\left(x_{n}^{2}-2 k\right)}\right]
$$

for all $k=1, \cdots, n$. From these inequalities, it follows that

$$
w_{k}\left(x_{n}\right)=\frac{x_{n}+\sqrt{x_{n}^{2}-2 k}}{2}\left[1+\frac{1}{2\left(x_{n}^{2}-2 k\right)}+O\left(n^{-2}\right)\right]
$$

as $n \rightarrow \infty$, uniformly in $k=1, \cdots, n$. By using a continuity argument, it can be shown that the validity of this asymptotic formula can be extended to complex $y \in \mathbb{C} \backslash[-1,1]$. Recall that $x_{n}=\sqrt{2 n} y$. By the trapezoidal rule

$$
\frac{1}{n} \sum_{k=1}^{n} f(k / n)=\int_{0}^{1} f(t) d t+\frac{f(1)-f(0)}{2 n}+O\left(n^{-2}\right)
$$

we have

$$
\begin{aligned}
\frac{1}{n} \sum_{k=1}^{n} \log \frac{x_{n}+\sqrt{x_{n}^{2}-2 k}}{x_{n}+\sqrt{x_{n}^{2}-2 n}} & =\frac{1}{n} \sum_{k=1}^{n} \log \left(y+\sqrt{y^{2}-k / n}\right)-\log \left(y+\sqrt{y^{2}-1}\right) \\
& \sim \int_{0}^{1} \log \left(y+\sqrt{y^{2}-t}\right) d t+\frac{1}{2 n} \log \frac{y+\sqrt{y^{2}-1}}{2 y}-\log \left(y+\sqrt{y^{2}-1}\right) \\
& =y^{2}-1 / 2-y \sqrt{y^{2}-1}+\frac{1}{2 n} \log \frac{y+\sqrt{y^{2}-1}}{2 y}
\end{aligned}
$$

and

$$
\begin{aligned}
\sum_{k=1}^{n} \log \left[1+\frac{1}{2\left(x_{n}^{2}-2 k\right)}\right] & \sim \sum_{k=1}^{n} \frac{1}{2\left(x_{n}^{2}-2 k\right)}=\frac{1}{n} \sum_{k=1}^{n} \frac{1}{4\left(y^{2}-k / n\right)} \\
& \sim \int_{0}^{1} \frac{d t}{4\left(y^{2}-t\right)}=\frac{1}{4} \log \frac{y^{2}}{y^{2}-1}
\end{aligned}
$$

as $n \rightarrow \infty$. Applying the last two formulas and (3.5) to (3.4) yields

$$
\begin{aligned}
\pi_{n}\left(x_{n}\right) & \sim \prod_{k=1}^{n}\left[\frac{x_{n}+\sqrt{x_{n}^{2}-2 n}}{2}\right] \cdot \prod_{k=1}^{n}\left[\frac{x_{n}+\sqrt{x_{n}^{2}-2 k}}{x_{n}+\sqrt{x_{n}^{2}-2 n}}\right] \cdot \prod_{k=1}^{n}\left[1+\frac{1}{2\left(x_{n}^{2}-2 k\right)}\right] \\
& \sim\left(\frac{n}{2}\right)^{n / 2}\left(y+\sqrt{y^{2}-1}\right)^{n} \exp \left[n\left(y^{2}-1 / 2-y \sqrt{y^{2}-1}\right)\right]\left(\frac{y+\sqrt{y^{2}-1}}{2 y}\right)^{1 / 2}\left(\frac{y^{2}}{y^{2}-1}\right)^{1 / 4} \\
& \sim\left(\frac{n}{2 e}\right)^{n / 2} \exp \left\{n\left[y^{2}-y \sqrt{y^{2}-1}+\log \left(y+\sqrt{y^{2}-1}\right)\right]\right\}\left(\frac{y+\sqrt{y^{2}-1}}{2 \sqrt{y^{2}-1}}\right)^{1 / 2},
\end{aligned}
$$

thus proving (3.3). 
Theorem 3.2. Let $\delta>0$ be any fixed small number. For $y$ in a small complex neighborhood of $[-1+$ $\delta, 1-\delta]$, we have

$$
\pi_{n}(\sqrt{2 n} y) \sim\left(\frac{n}{2 e}\right)^{n / 2} \frac{e^{n y^{2}}}{\left(1-y^{2}\right)^{1 / 4}}\left\{\cos \left[n(\theta-\sin \theta \cos \theta)+\frac{\theta}{2}\right]+\sin \left[n(\theta-\sin \theta \cos \theta)+\frac{\theta}{2}\right]\right\}
$$

as $n \rightarrow \infty$, where $\theta=\theta(y):=\arccos y$.

To prove the above theorem, we need a lemma analogous to Lemma 1 in $[8$. For convenience, we use the notation

$$
y_{ \pm}:=\left(\frac{n}{n \pm 1}\right)^{1 / 2} y \sim y \mp \frac{y}{2 n}+\frac{3 y}{8 n^{2}} .
$$

Lemma 3.3. Let $\varphi(y)$ be any analytic function in a small complex neighborhood of $[\delta, 1-\delta]$, we have

$$
\cos \left[(n \pm 1) \varphi\left(y_{ \pm}\right)\right] \sim \cos (n \varphi)\left(\cos \lambda \mp \frac{\mu}{n} \sin \lambda\right) \mp \sin (n \varphi)\left(\sin \lambda \pm \frac{\mu}{n} \cos \lambda\right)
$$

and

$$
\sin \left[(n \pm 1) \varphi\left(y_{ \pm}\right)\right] \sim \sin (n \varphi)\left(\cos \lambda \mp \frac{\mu}{n} \sin \lambda\right) \pm \cos (n \varphi)\left(\sin \lambda \pm \frac{\mu}{n} \cos \lambda\right)
$$

as $n \rightarrow \infty$, where

$$
\lambda=\lambda(y):=\varphi(y)-\frac{y \varphi^{\prime}(y)}{2},
$$

and

$$
\mu=\mu(y):=-\frac{y \varphi^{\prime}(y)}{8}+\frac{y^{2} \varphi^{\prime \prime}(y)}{8} .
$$

Proof. From (3.9) we have

$$
\begin{aligned}
(n \pm 1) \varphi\left(y_{ \pm}\right) & \sim n\left(1 \pm \frac{1}{n}\right) \varphi\left(y \mp \frac{y}{2 n}+\frac{3 y}{8 n^{2}}\right) \\
& \sim n\left(1 \pm \frac{1}{n}\right)\left(\varphi \mp \frac{y \varphi^{\prime}}{2 n}+\frac{3 y \varphi^{\prime}}{8 n^{2}}+\frac{y^{2} \varphi^{\prime \prime}}{8 n^{2}}\right) \\
& \sim n\left(\varphi \pm \frac{\lambda}{n}+\frac{\mu}{n^{2}}\right)
\end{aligned}
$$

where $\varphi$ denotes $\varphi(y)$, and $\lambda$ and $\mu$ are given in (3.12) and (3.13). It then follows that

$$
\begin{aligned}
\cos \left[(n \pm 1) \varphi\left(y_{ \pm}\right)\right] & \sim \cos (n \varphi) \cos (\lambda \pm \mu / n) \mp \sin (n \varphi) \sin (\lambda \pm \mu / n) \\
& \sim \cos (n \varphi)\left(\cos \lambda \mp \frac{\mu}{n} \sin \lambda\right) \mp \sin (n \varphi)\left(\sin \lambda \pm \frac{\mu}{n} \cos \lambda\right) ; \\
\sin \left[(n \pm 1) \varphi\left(y_{ \pm}\right)\right] & \sim \sin (n \varphi) \cos (\lambda \pm \mu / n) \pm \cos (n \varphi) \sin (\lambda \pm \mu / n) \\
& \sim \sin (n \varphi)\left(\cos \lambda \mp \frac{\mu}{n} \sin \lambda\right) \pm \cos (n \varphi)\left(\sin \lambda \pm \frac{\mu}{n} \cos \lambda\right) .
\end{aligned}
$$

This proves the lemma.

Proof of Theorem 3.2. Define

$$
p_{n}(x):=[\Gamma(n / 2+1 / 2)]^{-1} \pi_{n}(x) .
$$


We make a change of variable $x=x_{n}:=\sqrt{2 n} y$. It is easily seen from (3.1) and (3.14) that

$$
\frac{\Gamma(n / 2+1 / 2) \sqrt{2 n}}{\Gamma(n / 2+1)} \cdot y p_{n}(\sqrt{2 n} y)=p_{n+1}(\sqrt{2 n} y)+p_{n-1}(\sqrt{2 n} y) .
$$

As in (2.19), we now assume

$$
p_{n}(\sqrt{2 n} y) \sim n^{\alpha}[r(y)]^{n}\{f(y) \cos [n \varphi(y)]+g(y) \sin [n \varphi(y)]\}
$$

as $n \rightarrow \infty$. First, we shall determine the constant $\alpha$ and the functions $r(y)$ and $\varphi(y)$ in (3.16). From (3.9) and (3.16), we have

$$
\begin{aligned}
p_{n \pm 1}(\sqrt{2 n} y) & =p_{n \pm 1}\left(\sqrt{2(n \pm 1)} y_{ \pm}\right) \\
& \sim(n \pm 1)^{\alpha}\left[r\left(y_{ \pm}\right)\right]^{n \pm 1}\left\{f\left(y_{ \pm}\right) \cos \left[(n \pm 1) \varphi\left(y_{ \pm}\right)\right]+g\left(y_{ \pm}\right) \sin \left[(n \pm 1) \varphi\left(y_{ \pm}\right)\right]\right\} .
\end{aligned}
$$

Moreover, it can be shown from (3.9) that

$$
\left[r\left(y_{ \pm}\right)\right]^{n \pm 1} \sim r^{n \pm 1} e^{\mp y r^{\prime} / 2 r}
$$

where $r=r(y)$. Applying (3.9), (3.10), (3.11) and (3.18) to (3.17) yields

$$
p_{n \pm 1}(\sqrt{2 n} y) \sim n^{\alpha} r^{n \pm 1} e^{\mp y r^{\prime} / 2 r}[(f \cos \lambda \pm g \sin \lambda) \cos (n \varphi)+(g \cos \lambda \mp f \sin \lambda) \sin (n \varphi)] .
$$

Here $f$ and $g$ stand for $f(y)$ and $g(y)$. By Stirling's formula [1, (6.1.37)] we have

$$
\frac{\Gamma(n / 2+1 / 2) \sqrt{n / 2}}{\Gamma(n / 2+1)} \sim 1-\frac{1}{4 n} .
$$

A combination of (3.15), (3.16), (3.19) and (3.20) implies

$$
\begin{aligned}
2 y[f \cos (n \varphi)+g \sin (n \varphi)] \sim & r e^{-y r^{\prime} / 2 r}[(f \cos \lambda+g \sin \lambda) \cos (n \varphi)+(g \cos \lambda-f \sin \lambda) \sin (n \varphi)] \\
& +r^{-1} e^{y r^{\prime} / 2 r}[(f \cos \lambda-g \sin \lambda) \cos (n \varphi)+(g \cos \lambda+f \sin \lambda) \sin (n \varphi)] .
\end{aligned}
$$

Comparing the coefficients of $\cos (n \varphi)$ and $\sin (n \varphi)$ on both sides of the last formula gives

$$
\begin{gathered}
2 y f=r e^{-y r^{\prime} / 2 r}(f \cos \lambda+g \sin \lambda)+r^{-1} e^{y r^{\prime} / 2 r}(f \cos \lambda-g \sin \lambda) ; \\
2 y g=r e^{-y r^{\prime} / 2 r}(g \cos \lambda-f \sin \lambda)+r^{-1} e^{y r^{\prime} / 2 r}(g \cos \lambda+f \sin \lambda) .
\end{gathered}
$$

Solving the above two equations, we obtain

$$
\cosh \left(\log r-\frac{y r^{\prime}}{2 r}\right) \cos \lambda=y, \quad \sinh \left(\log r-\frac{y r^{\prime}}{2 r}\right) \sin \lambda=0 .
$$

A solution is

$$
\log r-\frac{y r^{\prime}}{2 r}=0, \quad \cos \lambda=y .
$$

The first equation in (3.21) implies

$$
r=e^{c y^{2}}
$$

for some constant $c \in \mathbb{C}$. From (3.12) and (3.21), we have

$$
\varphi= \pm\left(\arccos y-y \sqrt{1-y^{2}}\right)+c^{\prime} y^{2}+2 k \pi
$$


for some constant $c^{\prime} \in \mathbb{C}$ and $k \in \mathbb{N}$. Without loss of generality, we may take $c^{\prime}=0$ and $k=0$. Hence,

$$
\varphi=\arccos y-y \sqrt{1-y^{2}} .
$$

Next, we are going to determine the functions $f$ and $g$ in (3.16). From (3.9) and (3.22) we obtain

$$
\left[r\left(y_{ \pm}\right)\right]^{n \pm 1}=[r(y)]^{n}
$$

and

$$
f\left(y_{ \pm}\right) \sim f \mp \frac{y f^{\prime}}{2 n}, \quad g\left(y_{ \pm}\right) \sim g \mp \frac{y g^{\prime}}{2 n},
$$

where $f=f(y)$ and $g=g(y)$. Applying (3.10), (3.11), (3.24) and (3.25) to (3.17) yields

$$
\begin{aligned}
\frac{p_{n \pm 1}(\sqrt{2 n} y)}{n^{\alpha} r^{n}} \sim & (f \cos \lambda \pm g \sin \lambda) \cos (n \varphi)+(g \cos \lambda \mp f \sin \lambda) \sin (n \varphi) \\
& +\frac{\cos (n \varphi)}{n}\left( \pm \alpha f \cos \lambda \mp \mu f \sin \lambda \mp \frac{y f^{\prime}}{2} \cos \lambda+\alpha g \sin \lambda+\mu g \cos \lambda-\frac{y g^{\prime}}{2} \sin \lambda\right) \\
& +\frac{\sin (n \varphi)}{n}\left( \pm \alpha g \cos \lambda \mp \mu g \sin \lambda \mp \frac{y g^{\prime}}{2} \cos \lambda-\alpha f \sin \lambda-\mu f \cos \lambda+\frac{y f^{\prime}}{2} \sin \lambda\right) .
\end{aligned}
$$

A combination of (3.15), (3.16), (3.20) and (3.26) gives

$$
\begin{aligned}
\left(1-\frac{1}{4 n}\right)[2 y f \cos (n \varphi)+2 y g \sin (n \varphi)] \sim & 2 f \cos \lambda \cos (n \varphi)+2 g \cos \lambda \sin (n \varphi) \\
& +\frac{\cos (n \varphi)}{n}\left(2 \alpha g \sin \lambda+2 \mu g \cos \lambda-y g^{\prime} \sin \lambda\right) \\
& +\frac{\sin (n \varphi)}{n}\left(-2 \alpha f \sin \lambda-2 \mu f \cos \lambda+y f^{\prime} \sin \lambda\right) .
\end{aligned}
$$

In view of the second equation in (3.21), we obtain by matching the coefficients in the last formula

$$
\begin{aligned}
& \frac{f}{2} \cos \lambda+2 \alpha g \sin \lambda+2 \mu g \cos \lambda-y g^{\prime} \sin \lambda=0 ; \\
& \frac{g}{2} \cos \lambda-2 \alpha f \sin \lambda-2 \mu f \cos \lambda+y f^{\prime} \sin \lambda=0 .
\end{aligned}
$$

Note from (3.12), (3.13) and (3.23) that

$$
\lambda=\arccos y, \quad \mu=\frac{y}{4 \sqrt{1-y^{2}}} .
$$

Hence, equations (3.27) and (3.28) can be written as

$$
\begin{aligned}
& f+\frac{4 \alpha g \sqrt{1-y^{2}}}{y}+\frac{y g}{\sqrt{1-y^{2}}}-2 g^{\prime} \sqrt{1-y^{2}}=0 \\
& g-\frac{4 \alpha f \sqrt{1-y^{2}}}{y}-\frac{y f}{\sqrt{1-y^{2}}}+2 f^{\prime} \sqrt{1-y^{2}}=0 .
\end{aligned}
$$

Set

$$
u:=y^{-2 \alpha}\left(1-y^{2}\right)^{1 / 4} f ; \quad v:=y^{-2 \alpha}\left(1-y^{2}\right)^{1 / 4} g .
$$


We then have from (3.29), (3.30) and (3.31)

$$
u^{\prime}=-\frac{v}{2 \sqrt{1-y^{2}}} ; \quad v^{\prime}=\frac{u}{2 \sqrt{1-y^{2}}} .
$$

Define

$$
\theta=\theta(y):=\arccos y
$$

The solution of the system (3.32) is given by

$$
u=C_{1} \cos \frac{\theta}{2}+C_{2} \sin \frac{\theta}{2} ; \quad v=-C_{1} \sin \frac{\theta}{2}+C_{2} \cos \frac{\theta}{2},
$$

where $C_{1} \in \mathbb{C}$ and $C_{2} \in \mathbb{C}$ are two arbitrary constants. Consequently, we obtain from (3.31) that

$$
\begin{aligned}
& f=\frac{y^{2 \alpha}}{\left(1-y^{2}\right)^{1 / 4}}\left(C_{1} \cos \frac{\theta}{2}+C_{2} \sin \frac{\theta}{2}\right) ; \\
& g=\frac{y^{2 \alpha}}{\left(1-y^{2}\right)^{1 / 4}}\left(-C_{1} \sin \frac{\theta}{2}+C_{2} \cos \frac{\theta}{2}\right) .
\end{aligned}
$$

Applying (3.22), (3.35) and (3.36) to (3.16) yields

$$
p_{n}(\sqrt{2 n} y) \sim n^{\alpha} e^{n c y^{2}} y^{2 \alpha}\left(1-y^{2}\right)^{-1 / 4}\left[C_{1} \cos (n \varphi+\theta / 2)+C_{2} \sin (n \varphi+\theta / 2)\right] .
$$

This formula holds uniformly for $y$ in a small complex neighborhood of $[-1+\delta, 1-\delta]$. Moreover, it follows from (3.3) and (3.14) that

$$
p_{n}(\sqrt{2 n} y) \sim \frac{1}{\sqrt{2 \pi}} \exp \left\{n\left[y^{2}-y \sqrt{y^{2}-1}+\log \left(y+\sqrt{y^{2}-1}\right)\right]\right\}\left(\frac{y+\sqrt{y^{2}-1}}{2 \sqrt{y^{2}-1}}\right)^{1 / 2}
$$

for complex $y$ bounded away from $[-1,1]$. Finally, we match the above two formulas in an overlapping region to determine the constants $\alpha, c, C_{1}$ and $C_{2}$ in (3.37). For $\operatorname{Im} y>0$, it follows from (3.33) that $\operatorname{Im} \theta<0$; see a similar statement following (2.28). Furthermore, it can be shown from (3.23) that if $\operatorname{Im} y>0$, then we also have $\operatorname{Im} \varphi<0$. [To do this, one first notes that $\varphi^{\prime}(y)$ is negative for $y \in[-1+\delta, 1-\delta]$. Then, by the continuity of $\varphi^{\prime}$, one concludes that $\operatorname{Re} \varphi^{\prime}(y)<0$ for $y$ in a neighborhood of $[-1+\delta, 1-\delta]$ in the complex plane. Finally, the mean value theorem ensures that there exists a real number $\xi \in(0, \operatorname{Im} y)$ such that $\varphi(y)=\varphi(\operatorname{Re} y)+i(\operatorname{Im} y) \varphi^{\prime}(\operatorname{Re} y+i \xi)$, from which one obtains $\operatorname{Im} \varphi(y)<0$.] Thus, (3.37) implies

$$
p_{n}(\sqrt{2 n} y) \sim n^{\alpha} e^{n c y^{2}} y^{2 \alpha}\left(1-y^{2}\right)^{-1 / 4}\left(\frac{C_{1}}{2}+\frac{C_{2}}{2 i}\right) e^{i n \varphi+i \theta / 2} .
$$

Meanwhile, we have from (3.33) and (3.38)

$$
p_{n}(\sqrt{2 n} y) \sim \frac{1}{\sqrt{2 \pi}} \exp \left\{n\left[y^{2}-i y \sqrt{1-y^{2}}+i \arccos y\right]\right\}\left[\frac{e^{i(\theta-\pi / 2)}}{2 \sqrt{1-y^{2}}}\right]^{1 / 2} .
$$

Thus, we obtain from (3.23) and the above two formulas that $\alpha=0, c=1$ and

$$
\frac{C_{1}}{2}+\frac{C_{2}}{2 i}=\frac{e^{-i \pi / 4}}{2 \sqrt{\pi}}
$$

Similarly, matching (3.37) with (3.38) in the region $\operatorname{Im} y<0$ yields again $\alpha=0, c=1$ and the equation

$$
\frac{C_{1}}{2}-\frac{C_{2}}{2 i}=\frac{e^{i \pi / 4}}{2 \sqrt{\pi}}
$$


Coupling the last two equations gives

$$
C_{1}=C_{2}=\frac{1}{\sqrt{2 \pi}}
$$

Therefore, we conclude that

$$
\alpha=0, \quad c=1, \quad C_{1}=C_{2}=\frac{1}{\sqrt{2 \pi}} .
$$

This together with (3.14), (3.23) and (3.37) yields (3.8).

\section{Case 3: an open problem}

Recently, M. E. H. Ismail proposed the problem of finding asymptotic formulas for the orthogonal polynomials determined by

$$
\begin{aligned}
& \pi_{n+1}(x)=\left(x-n^{2}\right) \pi_{n}(x)-\frac{1}{4} \pi_{n-1}(x), \quad n \geq 1 \\
& \pi_{0}(x)=1, \quad \pi_{1}(x)=x
\end{aligned}
$$

see [5, §6] and [4, p. 370]. We first present a result for $x$ not in the interval of oscillation.

Theorem 4.1. As $n \rightarrow \infty$, we have

$$
\pi_{n}\left(n^{2} y\right) \sim\left(\frac{n}{e}\right)^{2 n} \exp \{n[(\sqrt{y}+1) \log (\sqrt{y}+1)-(\sqrt{y}-1) \log (\sqrt{y}-1)]\}\left(\frac{y}{y-1}\right)^{1 / 2}
$$

for complex y bounded away from $[0,1]$.

Proof. Set

$$
\pi_{n}(x)=\prod_{k=1}^{n} w_{k}(x)
$$

It follows from (4.1) and (4.2) that $w_{1}(x)=x$ and

$$
w_{k+1}(x)=x-k^{2}-\frac{1}{4 w_{k}(x)} .
$$

Let $x=x_{n}:=n^{2} y$ with $y \in \mathbb{C} \backslash[0,1]$. As with the case of Hermite polynomials, it can be shown that for real $x$ and $x \notin\left[0, n^{2}\right]$, we have

$$
x-(k-1)^{2}-1<w_{k}(x)<x-(k-1)^{2}+1
$$

for all $k=1, \cdots, n$. Thus,

$$
1+\frac{2 k}{x-k^{2}}-\frac{2}{x-k^{2}}<\frac{w_{k}(x)}{x-k^{2}}<1+\frac{2 k}{x-k^{2}}
$$

Consequently,

$$
w_{k}\left(n^{2} y\right)=n^{2}\left(y-\frac{k^{2}}{n^{2}}\right)\left[1+\frac{2 k}{n^{2} y-k^{2}}+O\left(n^{-2}\right)\right]
$$

as $n \rightarrow \infty$, uniformly in $k=1, \cdots, n$. By using a continuity argument, it can be shown that the validity of this asymptotic formula can be extended to complex $y \in \mathbb{C} \backslash[0,1]$. In view of the trapezoidal rule

$$
\frac{1}{n} \sum_{k=1}^{n} f(k / n) \sim \int_{0}^{1} f(t) d t+\frac{f(1)-f(0)}{2 n}
$$


we have

$$
\begin{aligned}
\sum_{k=1}^{n} \log \left(y-\frac{k^{2}}{n^{2}}\right) & \sim n \int_{0}^{1} \log \left(y-t^{2}\right) d t+\frac{1}{2} \log \frac{y-1}{y} \\
& =n[(\sqrt{y}+1) \log (\sqrt{y}+1)-(\sqrt{y}-1) \log (\sqrt{y}-1)-2]+\frac{1}{2} \log \frac{y-1}{y}
\end{aligned}
$$

and

$$
\sum_{k=1}^{n} \log \left(1+\frac{2 k}{n^{2} y-k^{2}}\right) \sim \sum_{k=1}^{n} \frac{2 k}{n^{2} y-k^{2}} \sim \int_{0}^{1} \frac{2 t}{y-t^{2}} d t=\log \frac{y}{y-1}
$$

as $n \rightarrow \infty$. Applying the last two formulas and (4.5) to (4.4) gives (4.3).

Next we give a result for $x$ inside the interval of oscillation.

Theorem 4.2. Let $\delta>0$ be any fixed small number. For $y$ in a small neighborhood of $[\delta, 1-\delta]$ in the complex plane, we have

$$
\pi_{n}\left(n^{2} y\right) \sim(-1)^{n-1} 2 \sin (n \pi \sqrt{y})\left(\frac{n}{e}\right)^{2 n}\left(\frac{1+\sqrt{y}}{1-\sqrt{y}}\right)^{n \sqrt{y}} y^{1 / 2}(1-y)^{n-1 / 2}
$$

as $n \rightarrow \infty$.

To prove the above theorem, we will need a lemma analogous to Lemma 1 in [8]. As in (3.9), for convenience we set

$$
y_{ \pm}:=\left(\frac{n}{n \pm 1}\right)^{2} y \sim y \mp \frac{2 y}{n}+\frac{3 y}{n^{2}}
$$

Lemma 4.3. Let $\varphi(y)$ be any analytic function in a small neighborhood of $[\delta, 1-\delta]$ in the complex plane, we have

$$
\cos \left[(n \pm 1) \varphi\left(y_{ \pm}\right)\right] \sim \cos (n \varphi)\left(\cos \lambda \mp \frac{\mu}{n} \sin \lambda\right) \mp \sin (n \varphi)\left(\sin \lambda \pm \frac{\mu}{n} \cos \lambda\right)
$$

and

$$
\sin \left[(n \pm 1) \varphi\left(y_{ \pm}\right)\right] \sim \sin (n \varphi)\left(\cos \lambda \mp \frac{\mu}{n} \sin \lambda\right) \pm \cos (n \varphi)\left(\sin \lambda \pm \frac{\mu}{n} \cos \lambda\right)
$$

as $n \rightarrow \infty$, where

$$
\lambda=\lambda(y):=\varphi(y)-2 y \varphi^{\prime}(y)
$$

and

$$
\mu=\mu(y):=y \varphi^{\prime}(y)+2 y^{2} \varphi^{\prime \prime}(y)
$$

Proof. From (4.7) we have

$$
\begin{aligned}
(n \pm 1) \varphi\left(y_{ \pm}\right) & \sim n\left(1 \pm \frac{1}{n}\right) \varphi\left(y \mp \frac{2 y}{n}+\frac{3 y}{n^{2}}\right) \\
& \sim n\left(1 \pm \frac{1}{n}\right)\left(\varphi \mp \frac{2 y \varphi^{\prime}}{n}+\frac{3 y \varphi^{\prime}}{n^{2}}+\frac{2 y^{2} \varphi^{\prime \prime}}{n^{2}}\right) \\
& \sim n\left(\varphi \pm \frac{\lambda}{n}+\frac{\mu}{n^{2}}\right)
\end{aligned}
$$


where $\lambda$ and $\mu$ are given in (4.10) and (4.11). It then follows that

$$
\begin{aligned}
\cos \left[(n \pm 1) \varphi\left(y_{ \pm}\right)\right] & \sim \cos (n \varphi) \cos (\lambda \pm \mu / n) \mp \sin (n \varphi) \sin (\lambda \pm \mu / n) \\
& \sim \cos (n \varphi)\left(\cos \lambda \mp \frac{\mu}{n} \sin \lambda\right) \mp \sin (n \varphi)\left(\sin \lambda \pm \frac{\mu}{n} \cos \lambda\right) \\
\sin \left[(n \pm 1) \varphi\left(y_{ \pm}\right)\right] & \sim \sin (n \varphi) \cos (\lambda \pm \mu / n) \pm \cos (n \varphi) \sin (\lambda \pm \mu / n) \\
& \sim \sin (n \varphi)\left(\cos \lambda \mp \frac{\mu}{n} \sin \lambda\right) \pm \cos (n \varphi)\left(\sin \lambda \pm \frac{\mu}{n} \cos \lambda\right) .
\end{aligned}
$$

This proves the lemma.

Proof of Theorem 4.2. Define

$$
p_{n}(x):=\frac{(-1)^{n}}{\Gamma(n)^{2}} \pi_{n}(x)
$$

We make a change of variable $x=x_{n}:=n^{2} y$. It is readily seen from (4.1) and (4.12) that

$$
(1-y) p_{n}\left(n^{2} y\right)=p_{n+1}\left(n^{2} y\right)+\frac{1}{4 n^{2}(n-1)^{2}} p_{n-1}\left(n^{2} y\right)
$$

As in (3.16), we first assume

$$
p_{n}\left(n^{2} y\right) \sim n^{\alpha}[r(y)]^{n}\{f(y) \cos [n \varphi(y)]+g(y) \sin [n \varphi(y)]\}
$$

as $n \rightarrow \infty$, and then determine the constant $\alpha$ and the functions $r(y), f(y), g(y)$ and $\varphi(y)$ in the formula. From (4.7) and (4.14) we have

$$
\begin{aligned}
p_{n \pm 1}\left(n^{2} y\right) & =p_{n \pm 1}\left((n \pm 1)^{2} y_{ \pm}\right) \\
& \sim(n \pm 1)^{\alpha}\left[r\left(y_{ \pm}\right)\right]^{n \pm 1}\left\{f\left(y_{ \pm}\right) \cos \left[(n \pm 1) \varphi\left(y_{ \pm}\right)\right]+g\left(y_{ \pm}\right) \sin \left[(n \pm 1) \varphi\left(y_{ \pm}\right)\right]\right\} .
\end{aligned}
$$

Moreover, it can be shown from (4.7) that as $n \rightarrow \infty$, we also have

$$
\left[r\left(y_{ \pm}\right)\right]^{n \pm 1} \sim r^{n \pm 1} e^{\mp 2 y r^{\prime} / r},
$$

where $r$ stands for $r(y)$. Applying (4.7), (4.8), (4.9) and (4.16) to (4.15) yields

$$
p_{n \pm 1}\left(n^{2} y\right) \sim n^{\alpha} r^{n \pm 1} e^{\mp 2 y r^{\prime} / r}[(f \cos \lambda \pm g \sin \lambda) \cos (n \varphi)+(g \cos \lambda \mp f \sin \lambda) \sin (n \varphi)] .
$$

A combination of (4.13), (4.14) and (4.17) gives

$$
(1-y)[f \cos (n \varphi)+g \sin (n \varphi)] \sim r e^{-2 y r^{\prime} / r}[(f \cos \lambda+g \sin \lambda) \cos (n \varphi)+(g \cos \lambda-f \sin \lambda) \sin (n \varphi)] .
$$

By comparing the coefficients of $\cos (n \varphi)$ and $\sin (n \varphi)$ on both sides of the last formula, we obtain

$$
\begin{gathered}
(1-y) f=r e^{-2 y r^{\prime} / r}(f \cos \lambda+g \sin \lambda) \\
(1-y) g=r e^{-2 y r^{\prime} / r}(g \cos \lambda-f \sin \lambda) .
\end{gathered}
$$

Thus, we have from the above equations

$$
(1-y)=r e^{-2 y r^{\prime} / r} \cos \lambda, \quad 0=r e^{-2 y r^{\prime} / r} \sin \lambda .
$$

The only solution is $\lambda=0$, and

$$
r e^{-2 y r^{\prime} / r}=1-y
$$


With $\lambda=0$, we obtain from (4.10)

$$
\varphi=c \sqrt{y}
$$

for some constant $c \in \mathbb{C}$. Let $R(y):=\log r(y)$. From (4.18), it is easily seen that $R(y)$ satisfies a first-order linear inhomogeneous equation, whose solution is given by

$$
R(y)=-\frac{1}{2} y^{1 / 2}\left[\int^{y} s^{-3 / 2} \log (1-s) d s\right] .
$$

Upon integration by parts, followed by a change of variable $u=s^{1 / 2}$, one obtains

$$
R(y)=\log (1-y)+2 y^{1 / 2} \operatorname{arctanh} \sqrt{y}+c^{\prime} \sqrt{y}
$$

for some constant $c^{\prime} \in \mathbb{C}$. Taking exponential on both sides gives

$$
r(y)=(1-y)\left(\frac{1+\sqrt{y}}{1-\sqrt{y}}\right)^{\sqrt{y}} e^{c^{\prime} \sqrt{y}} .
$$

Without loss of generality, we may assume $c^{\prime}=0$. Hence,

$$
r(y)=(1-y)\left(\frac{1+\sqrt{y}}{1-\sqrt{y}}\right)^{\sqrt{y}}
$$

Next we determine the functions $f$ and $g$ in (4.14). From (4.7), (4.18) and (4.20) we have

$$
\left[r\left(y_{ \pm}\right)\right]^{n \pm 1} \sim(1-y)^{ \pm 1}[r(y)]^{n}\left[1+\frac{y}{n(1-y)}\right] .
$$

Furthermore, it is easily seen from (4.7) and (4.19) that $(n \pm 1) \varphi\left(y_{ \pm}\right) \sim n \varphi(y)$ and

$$
f\left(y_{ \pm}\right) \sim f(y) \mp \frac{2 y f^{\prime}(y)}{n}, \quad g\left(y_{ \pm}\right) \sim g(y) \mp \frac{2 y g^{\prime}(y)}{n} .
$$

Applying the above formulas for functions $r, \varphi, f$ and $g$ to (4.15) yields

$$
p_{n \pm 1}\left(n^{2} y\right) \sim n^{\alpha} r^{n}(1-y)^{ \pm 1}\left(1 \pm \frac{\alpha}{n}\right)\left[1+\frac{y}{n(1-y)}\right]\left[\left(f \mp \frac{2 y f^{\prime}}{n}\right) \cos (n \varphi)+\left(g \mp \frac{2 y g^{\prime}}{n}\right) \sin (n \varphi)\right] .
$$

[One can also obtain this result from Lemma 4.3, since $\lambda=\mu=0$ by (4.19).] This together with (4.13) and (4.14) implies

$f \cos (n \varphi)+g \sin (n \varphi) \sim\left[f+\frac{1}{n}\left(\frac{y f}{1-y}+\alpha f-2 y f^{\prime}\right)\right] \cos (n \varphi)+\left[g+\frac{1}{n}\left(\frac{y g}{1-y}+\alpha g-2 y g^{\prime}\right)\right] \sin (n \varphi)$.

Comparing the coefficients on both sides of the last formula gives

$$
\frac{y f}{1-y}+\alpha f-2 y f^{\prime}=0, \quad \frac{y g}{1-y}+\alpha g-2 y g^{\prime}=0
$$

Hence,

$$
f=C_{1} y^{\alpha / 2}(1-y)^{-1 / 2}, \quad g=C_{2} y^{\alpha / 2}(1-y)^{-1 / 2},
$$

where $C_{1} \in \mathbb{C}$ and $C_{2} \in \mathbb{C}$ are two arbitrary constants. Applying (4.19), (4.20) and (4.22) to (4.14) yields

$$
p_{n}\left(n^{2} y\right) \sim n^{\alpha} y^{\alpha / 2}(1-y)^{n-1 / 2}\left(\frac{1+\sqrt{y}}{1-\sqrt{y}}\right)^{n \sqrt{y}}\left[C_{1} \cos (n c \sqrt{y})+C_{2} \sin (n c \sqrt{y})\right] .
$$


This formula holds uniformly for $y$ in a small neighborhood of $[\delta, 1-\delta]$ in the complex plane. Moreover, it follows from (4.3) and (4.12) that

$$
p_{n}\left(n^{2} y\right) \sim \frac{(-1)^{n} n}{2 \pi} \exp \{n[(\sqrt{y}+1) \log (\sqrt{y}+1)-(\sqrt{y}-1) \log (\sqrt{y}-1)]\}\left(\frac{y}{y-1}\right)^{1 / 2}
$$

for complex $y$ bounded away from $[0,1]$. At the final stage, we match the last two formulas in an overlapping region to determine the constants $\alpha, c, C_{1}$ and $C_{2}$ in (4.23). In view of the equalities $\exp ( \pm i n c \sqrt{y})=\cos (n c \sqrt{y}) \pm i \sin (n c \sqrt{y})$ and

$$
(1-y)^{n}\left(\frac{1+\sqrt{y}}{1-\sqrt{y}}\right)^{n \sqrt{y}}=\exp \{n[(\sqrt{y}+1) \log (\sqrt{y}+1)-(\sqrt{y}-1) \log (1-\sqrt{y})]\},
$$

formula (4.23) can be written as

$$
\begin{aligned}
p_{n}\left(n^{2} y\right) \sim & n^{\alpha} y^{\alpha / 2}(1-y)^{-1 / 2} \exp \{n[(\sqrt{y}+1) \log (\sqrt{y}+1)-(\sqrt{y}-1) \log (1-\sqrt{y})]\} \\
& \times\left[\left(\frac{C_{1}}{2}-\frac{C_{2}}{2 i}\right) e^{-i n c \sqrt{y}}+\left(\frac{C_{1}}{2}+\frac{C_{2}}{2 i}\right) e^{i n c \sqrt{y}}\right] .
\end{aligned}
$$

Meanwhile, it follows from (4.24) that for $\operatorname{Im} y>0$, we have

$$
p_{n}\left(n^{2} y\right) \sim \frac{n}{2 \pi} \exp \{n[(\sqrt{y}+1) \log (\sqrt{y}+1)-(\sqrt{y}-1) \log (1-\sqrt{y})]-i n \pi \sqrt{y}-i \pi / 2\}\left(\frac{y}{1-y}\right)^{1 / 2} .
$$

A comparison of the above two asymptotic formulas shows that $\alpha=1$ and $c=\pi$ or $c=-\pi$. Without loss of generality, we take $c=\pi$. Note that the function $\exp (i n c \sqrt{y})=\exp (i n \pi \sqrt{y})$ is exponentially small, and hence negligible in the region $\operatorname{Im} y>0$. By matching the last two formulas one more time, and ignoring the exponentially small term, we have

$$
\frac{C_{1}}{2}-\frac{C_{2}}{2 i}=\frac{e^{-i \pi / 2}}{2 \pi}
$$

With $\alpha=1$ and $c=\pi$, we match (4.24) with (4.25) in the region $\operatorname{Im} y<0$ to obtain the other equation

$$
\frac{C_{1}}{2}+\frac{C_{2}}{2 i}=\frac{e^{i \pi / 2}}{2 \pi} .
$$

Upon solving the last two equations, we obtain $C_{1}=0$ and $C_{2}=-1 / \pi$. Therefore, we conclude that

$$
\alpha=1, \quad c=\pi, \quad C_{1}=0, \quad C_{2}=-1 / \pi .
$$

Combining this with (4.12) and (4.23) gives (4.6).

\section{References}

[1] M. Abramowitz and I. A. Stegun, "Handbook of Mathematical Functions, with Formulas, Graphs, and Mathematical Tables", Dover Publications, Inc., New York, 1970.

[2] P. Deift, T. Kriecherbauer, K. T.-R. McLaughlin, S. Venakides and X. Zhou, Uniform asymptotics for polynomials orthogonal with respect to varying exponential weights and applications to universality questions in random matrix theory, Comm. Pure Appl. Math. 52 (1999), 1335-1425.

[3] P. Deift, T. Kriecherbauer, K. T.-R. McLaughlin, S. Venakides and X. Zhou, Strong asymptotics of orthogonal polynomials with respect to exponential weights, Comm. Pure Appl. Math. 52 (1999), 1491-1552. 
[4] S. S. Goh and C. A. Micchelli, Uncertainty principles in Hilbert spaces, J. Fourier Anal. Appl. 8 (2002), 335-373.

[5] M. E. H. Ismail and E. Koelink, The J-Matrix method and eigenfunction expansions, preprint (July, 2010).

[6] R. Koekoek and R. F. Swarttouw, "The Askey-scheme of Hypergeometric Orthogonal Polynomials and its q-analogue", Report no. 98-17, TU-Delft, 1998.

[7] F. W. J. Olver, "Asymptotics and Special Functions", Academic Press, New York, 1974. Reprinted by A. K. Peters, Wellesley, MA, 1997.

[8] Z. Wang and R. Wong, Uniform asymptotic expansion of $J_{\nu}(\nu a)$ via a difference equation, Numer. Math. 91 (2002) 147-193.

[9] R. Wong, "Asymptotic Approximations of Integrals", Academic Press, Boston, 1989. (Reprinted by SIAM, Philadelphia, PA, 2001.)

[10] R. Wong and H. Li, Asymptotic expansions for second-order linear difference equations, J. Comput. Appl. Math. 41 (1992) 65-94. 